Gesnerus 55 (1998) 87-116

\title{
Eugen Bleulers Warnung vor dem «autistisch- undisziplinierten Denken in der Medizin» als Beitrag zur Erkenntniskritik ärztlicher Forschung
}

Ein Zürcher Konzept über «ratio et experientia» im ärztlichen Denken ${ }^{1}$.

Caroline Jagella

\section{Summary}

Bleuler, well known for introducing psychoanalysis into university psychiatry, is less known for his more important achievement: his introduction of results of brain research into scientific thinking, especially epistemology. His short tract on "autistic and undisciplined thinking" summarises these themes, culminating in his critical rejection of a science which denies the "natural unity" of the brain, and insists on the separation of emotions from thinking despite the evidence to the contrary gained from studying associations and their physical substrate.

1 Friedrich Hoffmann (1660-1742), einer der einflussreichsten Ärzte des 18. Jahrhunderts, hatte die Begriffe «ratio et experientia» in seiner «Medicina rationalis systematica» (Halle 1718-1740) einander in neuartiger Weise gegenübergestellt. Im ersten Band (neue Auflage von 1739) heisst es dazu: «Damit wir aber diese überaus nützliche Lehre uns aneignen, sind zwei Hilfsmittel erforderlich, die die alleinigen und eigentlichen Fundamente der Wahrheit und Gewissheit in der Heilkunst sind: deren eines heisst Experientia (Erfahrung), das andere Ratio (Überlegung, Vernunft)» (S. 8). «Unter Experientia wollen wir nicht jene verwegene und trügerische Erfahrung der Unkundigen verstanden haben, die geradewegs die Ursache günstiger und ungünstiger, nach Einnahme eines Mittels beobachtete Wirkungen dem gegebenen Mittel zuschreiben, ohne auf das Individuum oder andere Umstände und Ursachen Rücksicht zu nehmen.» «Unter Ratio wollen wir nicht so sehr das richtige Urteil des Arztes verstanden wissen, oder die geschulte Fähigkeit, richtig Schlüsse zu ziehen, die überall und immer, wo die Wahrheit ermittelt wird, erforderlich ist, als vielmehr die wahre medizinische Theorie, und zwar die reale, nicht die nominale der Scholastiker» (S. 9f.; Übersetzung der Zitate von Prof. Dr. med. Urs Boschung, Bern).

Dr. med. des. Caroline Jagella, Medizinhistorisches Institut, Rämistrasse 71, 8006 Zürich 


\section{Zusammenfassung}

Eugen Bleuler ist vor allem dafür bekannt, dass er die Psychoanalyse in die Universitätspsychiatrie eingeführt hat. Eine weitgehend vernachlässigte, langfristig gesehen aber bedeutungsvollere Leistung Bleulers liegt in seinem Versuch, die Ergebnisse der Hirnforschung für das wissenschaftliche Denken, die Erkenntnistheorie der Naturwissenschaft nutzbar zu machen. Die kurze Abhandlung über das «autistisch-undisziplinierte Denken in der Medizin» fasst diesen Aspekt seiner Forschung zusammen und spiegelt seine kritische Ablehnung einer Wissenschaft, die die «natürliche Einheit» des Gehirns leugne. Eine Naturwissenschaft, die darauf bestehe, Gefühle vom Denken zu trennen, obwohl die Forschungen über Assoziationen und ihr physisches Substrat ganz offensichtlich das Gegenteil gezeigt hätten, nehme sich die Chance, ihr eigenes Vorgehen kritisch und fruchtbringend zu überdenken.

\section{Einleitung}

«... und die höchste Erkenntnis ist nicht die, dass man nichts weiss, sondern die, dass man unterscheidet zwischen dem Bekannten und dem Unbekannten, dass man weiss, was man weiss und was man nicht weiss.»²

Die Frage, wo die Grenze zwischen Wissen und Noch-nicht-Wissen liegt, ist für die Medizin keine akademische Angelegenheit. Theoretische Reflexionen über mögliche Erweiterungen der Erkenntnismöglichkeit tragen nur dann, wenn sie in irgendeiner Weise an der Praxis geprüft werden können, in die für alle nachvollziehbare Effizienz praktischer Handlungsweise transponiert werden können. An diesem Punkt unterscheidet sich die Medizin von anderen akademischen Disziplinen, von denen sie umgekehrt vornehmlich aus diesem Grunde, der Heterogenität ihrer Ansprüche, auch immer wieder als Nicht-Wissenschaft betrachtet wurde und wird. Alle Bemühungen, die einzelnen Spezialfächer möglichst rein naturwissenschaftlich zu präsentieren, scheitern potentiell an diesem Moment praktischer Bewährung. Denn die Definition als medizinische Disziplin impliziert mit wenigen Ausnahmen bis heute den Anspruch, Menschen helfen zu wollen. Die Erkenntnistheorie der Medizin, sofern man überhaupt von einer solcher sprechen will, entsteht

2 Bleuler, Eugen: Das autistisch-undisziplinierte Denken in der Medizin und seine Überwindung. Berlin, Springer, 1919, S. 90. 
aus dieser Spannung zwischen wissenschaftlichem und ethischem Anspruch, kann also nie «rein» sein. Das medizinische Denken ist nie losgelöst von der Erfahrung täglicher Praxis. Dadurch wird die Vielfalt der zu berücksichtigenden Faktoren jedoch nahezu unendlich, und die Reduktion auf fassbare Übereinstimmungen oder Differenzen wird fragwürdig, wenn man sie als absolut annimmt. Die Spannung Naturwissenschaft versus Anwendbarkeit findet sich auf Seite der Handelnden wieder in dem Konflikt Forscher versus Arzt, der bis heute die medizinische Diskussion beherrscht.

Eugen Bleuler (1857-1939) sah die Medizin seiner Zeit in einem eigentümlichen Spannungsverhältnis befangen, in dem sich der enorme Fortschritt in den Naturwissenschaften einerseits und irrationales Verhalten der Ärzte andererseits zu einem scheinbar unüberbrückbaren Gegensatz aufbauten. "Das autistisch-undisziplinierte Denken in der Medizin und seine Überwindung» erschien 1919, im Jahr nach dem Ende des Ersten Weltkriegs, und es ist ohne diesen Zeitbezug nicht verstehbar. Die gesellschaftliche Krise war mit einer Krise der Medizin aufs engste verknüpft. Die Frage, ob und wie die Medizin eine Wissenschaft sein kann, wurde zu einer typischen Frage des 20. Jahrhunderts.

Mit der Hirnforschung des späten 19. Jahrhunderts war ein neues Moment in die erkenntnistheoretische Diskussion gekommen, das in seinen Konsequenzen für die Naturwissenschaft ähnlich grundlegend war, wie die Thesen Kants hundert Jahre zuvor. Das Gehirn als das Organ, das Wissenschaft denkt, aber genauso Kultur und Politik gestaltet, konnte und musste in den wissenschaftlichen Diskurs einbezogen werden. Positivistische Forschung hatte sich gegen dasjenige spekulative Element der Schellingschen Naturphilosophie gewandt, das die Intuition des Subjekts, also des Forschers, als Instrument der Erkenntnisfindung anerkannte. Johannes Müller (1801-1858), Johann Lucas Schönlein (1793-1864) und Rudolf Virchow (1821-1902) hatten ebenso philosophische Denkansätze wie praktische Methoden aus der Naturphilosophie der Romantik in die sogenannte positivistische Epoche der Medizin tradiert ${ }^{3}$. Dennoch wurde die SubjektObjekt-Problematik und ihre Konsequenzen für den Naturforscher selbst von ihren Schülern immer weniger diskutiert. Die Gehirn-oder-SeeleDiskussion des Materialismusstreits um 1860 hatte jedoch deutlich gemacht, dass sich auch die positivistische Medizin auf die Konfrontation mit traditionell philosophischen Domänen einstellen musste, ohne dass ihr eigenes

3 Lohff, Brigitte: Die Suche nach der Wissenschaftlichkeit der Physiologie in der Zeit der Romantik: ein Beitrag zur Erkenntnisphilosophie der Medizin. Stuttgart u. a., Fischer, 1990; Bleker, Johanna: Die Naturhistorische Schule 1825-1845. Stuttgart u. a., Fischer, 1981. 
Erscheinungsbild dadurch zunächst in Frage gestellt wurde. Emil Du BoisReymonds (1818-1896) «Ignorabimus» von 1872 «in Bezug auf das Räthsel ..., was Materie und Kraft seien» ${ }^{4}$ wurde als Grundmodell positivistischer Geisteshaltung interpretiert.

Bleulers Schrift stellt Erkenntniswert und Erkenntnissuche der Medizin auf zwei Ebenen in Frage, die aber beide die Subjekt-Objekt-Beziehung behandeln. Auf der ersten Ebene geht es um die Praxis des medizinischen Alltags, und sie erschliesst sich unmittelbar aus den Aussagen des Buchs. Auf einer zweiten Ebene wird die grundsätzliche Frage nach der Richtigkeit herkömmlicher positivistischer Erkenntnistheorie gestellt.

\section{Das Werk und der Autor}

Bereits aus dem Titel lässt sich erahnen, dass es sich um eine Streitschrift, auf jeden Fall aber um eine ganz ungewöhnliche und ungewohnte Arbeit handeln müsse: hier wird mit einem medizinischen Terminus - «autistisch» - die Medizin selbst kritisiert.

Eugen Bleuler befand sich zum Zeitpunkt dieser Veröffentlichung auf der Höhe seiner wissenschaftlichen Laufbahn. Acht Jahre zuvor war mit der «Dementia praecox oder Gruppe der Schizophrenien» eines der bedeutendsten und wegweisenden Werke der Psychiatriegeschichte erschienen. Bleuler hatte hierin den von ihm kreierten Begriff der «Schizophrenie» zum ersten Mal in einem grösseren Rahmen erläutert und damit einem breiten ärztlichen Publikum nahegebracht ${ }^{5}$. Sein "Lehrbuch der Psychiatrie» von 1916 wurde zum Standardwerk psychiatrischer Lehre des 20. Jahrhunderts 6 . Hier hatte er seine Thesen über die Ätiologie der Schizophrenie, ihre Abgrenzung von der von Emil Kraepelin (1856-1926) gefundenen Krankheitsbezeichnung der «Dementia praecox» und ihrer möglichen Therapie weiter ausgeformt und in den studentischen Unterricht integriert. Seit 1898 war Bleuler Direktor der Psychiatrischen Universitätsklinik Zürich auf dem Burghölzli und angesehener Professor für Psychiatrie an der Zürcher

4 Du Bois-Reymond, Emil: Über die Grenzen des Naturerkennens. 3. Auflage. Leipzig, Veit \& Comp., 1873, S. 33.

5 Bleuler, Eugen: Dementia praecox oder Gruppe der Schizophrenien. Leipzig u.a., Deuticke, 1911. Dass «Schizophrenie» schliesslich ein so populärer Begriff wurde, bis heute in allen möglichen nicht-psychiatrischen Bezügen verwendet, zeigt, wie genau Bleuler hier anscheinend ein Grundgefühl des 20. Jahrhunderts angesprochen und ausgedrückt hat.

6 Bleuler, Eugen: Lehrbuch der Psychiatrie. Berlin, Springer, 1916. 
Medizinischen Fakultät ${ }^{7}$. Dass diese Arbeit ihm ein besonderes Anliegen war, verrät ihr emotionaler Ton, der im Kontext seiner früheren Werke einen auffallenden Stilbruch bedeutet. Er verwertet hierin die Erfahrungen langjähriger Praxis zu einem Appell an die Vernunft seiner ärztlichen Kollegen.

Es ist der Blick des Psychiaters, den Bleuler hier auf die Medizin seiner Zeit richtet. Wenn er in bezug auf ärztliches Handeln immer wieder vom «Grössenwahn» oder «Verfolgungswahn» spricht, oder von «affektiven Bedürfnissen», so verwendet er psychiatrische Terminologie. Hierin lag dann auch das Skandalträchtige des Buchs: jedem der ärztlichen Leser war zu diesem Zeitpunkt die Bedeutung des Terminus «autistisch» als ein Hauptsymptom der Schizophrenie bekannt, wie es Bleuler 1911 als erster beschrieben und benannt hatte ${ }^{8}$. Jedem der damaligen Kollegen wird bei dem Wort «autistisch» die fehlende oder stark eingeschränkte Fähigkeit zur Kontaktaufnahme mit der Aussenwelt im Sinne des Realitätsverlusts eingefallen sein. Bleuler wusste natürlich, dass seine Definition des Autismus als einer Denkform, die «sich um die Realität nicht kümmert»', zu allgemeiner Bekanntheit gelangt war $^{10}$. Bleuler hatte das autistische Denken als ein Denken charakterisiert, das von Affekten und Wunschvorstellungen geleitet wird, bei dem abgespalten wird, «was nicht passt», ein Denken, das zwischen innerer und äusserer Realität nicht mehr zu unterscheiden ver-

7 Eugen Bleuler, 1857 in Zollikon geboren, wuchs im ländlichen Milieu auf. Nach dem Medizinstudium in Zürich ging er zunächst als Assistenzarzt an die Psychiatrische Universitätsklinik Waldau (Bern). 1884/85 war er zu Studienaufenthalten in Paris, London und München. Die prägendste und für die Entstehung des Schizophreniekonzepts bedeutungsvollste Periode waren die zwölf Jahre von 1886-1898, in denen er als Direktor der Psychiatrischen Klinik Rheinau (Zürich) arbeitete. Die betont einfache, bäuerlich-ländliche Lebensweise dieser Jahre, in engster Nähe zu «seinen» Patienten wurde für Bleuler zur Referenz seines späteren Denkens und Arbeitens. Als Nachfolger August Forels war er von 1898 bis 1927 Direktor am Burghölzli.

8 Eine erste Abhandlung über «Die Prognose der Dementia praecox», die sich dem Schizophreniekonzept annähert, erschien bereits im Jahr 1908. «Das autistische Denken» war Inhalt eines Vortrags, den Bleuler auf der 83. Versammlung Deutscher Naturforscher und Ärzte in Karlsruhe vom 24. bis 29. September 1911 hielt. Vgl. auch: «Das autistische Denken», in: Jahrbuch für psychoanalytische Forschungen 4 (1912). Der Terminus «autistisch» gehört, wie auch «Affektivität» und «Ambivalenz», zu den heute gängigen Bezeichnungen, die von Bleuler eingeführt wurden.

9 Bleuler 1916, S. 33.

10 Bekannt war wohl auch folgende Passage: «Wo aber die Kranken sich ganz in ihren Autismus verpuppt haben, hat die Aussenwelt höchstens noch insofern Wirklichkeitswert, als sie sie in ihren Gedanken stört; wenn dann ein Affekt äussere Ereignisse begleitet, so kann es nur noch der der 'Ablehnung' sein. Die scheinbar parathymische Selbstzufriedenheit vieler Schizophrener ist von ihrem Standpunkt aus gar nicht abnorm, da im autistischen Denken ihre Wünsche erfüllt sind» (Bleuler 1911, S. 301). 
$\operatorname{mag}^{11}$. Das autistische Denken verliert «den Kontakt mit der Wirklichkeit», die Patienten leben in «einer eingebildeten Welt von allerlei Wunscherfüllungen und Verfolgungsideen». Angewandt auf die Realität der Medizin rührt das Wort «autistisch» an ein Privileg, das seit dem Aufkommen des Positivismus von dieser am meisten gehütet worden war: dem der Objektivität. «Autistisches Denken», wie es Bleuler definierte, lässt sich nicht vereinbaren mit den Kriterien der Objektivität, es ist vielmehr ein Denken, «das keine Rücksicht nimmt auf die Grenzen der Erfahrung, und das auf eine Kontrolle der Resultate an der Wirklichkeit und eine logische Kritik verzichtet» ${ }^{2}$. Es wird zum Widerpart des Erfahrbaren, des Realen, des Logischen - und damit aller Charakteristika, die die modernen Naturwissenschaften definieren. Im «Lehrbuch der Psychiatrie» war das autistische Denken 1916 von Bleuler als ein «pathologischer Zustand» beschrieben worden, der sich auszeichne durch «eine grosse Loslösung von der Erfahrung, die leicht zu Unsinn führt» ${ }^{13}$. Drei Jahre später war «autistisch» nicht nur zugkräftiges Schlagwort eines Titels, es wurde zum Leitmotiv einer ganzen Abhandlung, die die Medizin als Profession und Wissenschaft ins Visier nahm. Die "parathymische Selbstzufriedenheit»" ${ }^{14}$, die Bleuler bei den Schizophrenen festgestellt hatte, findet er auch bei den Ärzten - und, wie bei seinen psychiatrischen Patienten, sieht er den Grund hierfür in der irrigen Imaginierung erfüllter Wünsche.

Die Bedeutung des Wortes «autistisch» erfährt jedoch unterschiedliche Gewichtungen und bezeichnet nicht immer pathologische Verhaltensweisen. In diesen Nuancierungen verbirgt sich eine für Bleulers psychiatrisches Denken ungemein wichtige Überzeugung: dass es in der Terminologie von Krankheit keine absolute Sicht geben könne, sondern immer nur eine Annäherung ${ }^{15}$. Diese sollte dann allerdings das Resultat einer maximalen Berücksichtigung aller Umstände und Faktoren sein. Bleuler hat dieses Prinzip in seinem Schizophreniekonzept verwirklicht, das in erster Linie ein Erfahrungskonzept war $^{16}$. Dieses Verständnis, das auf ständiger kritischer

11 L. c.; vgl. Bleuler 1916, S. 33.

12 Bleuler 1919, S. 1.

13 Bleuler 1916, S. 16.

14 Bleuler 1919, S. 83; Parathymie meint einen der realen Situation oder dem Denkinhalt nicht entsprechenden, oft auch genau entgegengesetzten Affektzustand: para (gr.) im Sinne von neben; thymos (gr.) = Gemüt. Vgl. Bleuler 1911, S. 303.

15 Vgl. Bleuler 1916, S. 11; Bleuler 1911, S. 229.

16 Vgl. das Vorwort zu Bleuler 1911. Bleuler verstand die Bezeichnung «Schizophrenie» als «vorläufig», was für ihn aber nicht gleichbedeutend war mit «unklar». Im Kontext seiner Medizinkritik hielt er fest, dass die Begriffskonstruktion nicht schade, solange man sich ihrer Mangelhaftigkeit «bewusst» sei (Bleuler 1919, S. 59). 
Prüfung eigener Denk- und Vorgehensweise beruht, war es, das er bei seinen Kollegen vermisste.

\section{Der Ansatz der Kritik}

Parallel zum psychiatrischen Phänomen «autistisch» diagnostiziert Bleuler bei der Medizin seiner Zeit die Unfähigkeit, ihre Stellung innerhalb der Welt naturwissenschaftlicher Realität einzuschätzen. Die Medizin habe den Kontakt zur Aussenwelt des Nachvollziehbaren verloren und sich in ihre eigenen Postulate verstrickt, die sich oft im eklatanten Gegensatz zur Alltagserfahrung therapeutischer Erfolge respektive Misserfolge befänden. Die Forderungen nach möglichster Exaktheit hätten einen ungenügenden Einfluss auf das Denken und namentlich das Handeln der Ärzte ${ }^{17}$. Eine Art Bewusstseinsspaltung verhindere, dass vorhandene Erkenntnisse ihren Weg nach «aussen», ins ärztliche Handeln, fänden. «Autistisches Denken in der Medizin» sieht Bleuler als Folge der Unwägbarkeiten eines Fachs, das eigentlich nicht als Wissenschaft bezeichnet werden dürfe. Denn anders als in den exakten Wissenschaften sei in der Medizin weder die beliebige Herbeiführung bestimmter Situationen möglich, noch hätte man zur Überprüfung seiner Thesen beliebig viel Zeit zur Verfügung. «Instinktive» Bedürfnisse von Arzt und Patient entzögen sich logischer Bestimmung. In der Medizin herrsche «zum Unterschied von allen anderen Wissenschaften noch am meisten autistisches Denken ${ }^{18}$, weil hier die Diskrepanz zwischen dem Wunsch nach Erkenntnis und den tatsächlichen Möglichkeiten am allergrössten sei ${ }^{19}$. In dem Moment, wo das Subjekt des Arztes nicht mehr von der Situation getrennt werden könne, entstehe der Boden für autistisches Denken. Dieses Subjekt wiederum beginne immer da eine Rolle zu spielen, wo keine exakten Verhaltensmassregeln vorliegen, da, wo der Handlungsspielraum aufgrund mangelnden Wissens grösser wird. Das Ausmass des Mangels an Wissen sei mehr oder weniger zeitgebunden - nicht zeitgebunden sei hingegen die Fähigkeit des einzelnen Arztes zu erkennen, inwie-

17 Op. cit., S. III.

18 Op. cit., S. 2.

19 Wichtig ist die Unterscheidung zwischen einer bloss nachlässig-resignativen Haltung und der gerichteten Haltung, die das autistische Denken nach Bleuler ausmacht. Die Ausrichtung beim autistischen Denken ist unmittelbar mit einem Ziel, nämlich der Wunscherfüllung, gekoppelt und eben damit affektverbunden. Das muss betont werden, weil so deutlich wird, dass es sich hier um eine Kritik am wissenschaftlichen Vorgehen handelt und nicht um die Anprangerung menschlicher Nachlässigkeiten. 
weit bei der Erstellung einer Diagnose oder der Wahl einer Therapie persönliche, somit potentiell irrationale Gründe eine Rolle spielten.

Diese Vorwürfe beziehen sich vordergründig vor allem auf Situationen, die das tägliche Brot des praktischen Arztes ausmachen. Darüber hinaus spricht Bleuler hier aber eine Aufgabenteilung an, die damals mehr und mehr üblich zu werden begann: die Einteilung in "praktische Medizin» auf der einen Seite und in «theoretische Forschung» auf der anderen Seite. Wichtige Beobachtungen und Erfahrungen, die einem nur der ärztliche Alltag biete, würden viele Ärzte «in autistischer Weise von [ihren] medizinischen Überlegungen absperren, statt sie zur Basis [ihrer] therapeutischen Handlungen und Forschungen zu machen $»^{20}$.

Es handelt sich um ein komplexes Beziehungsgeflecht verschiedenster Beweggründe, aus dem autistische Handlungen im Bleulerschen Sinne entspringen können. Bei einer solchen Betrachtungsweise kann die Einbindung des einzelnen in die Gemeinschaft nicht unberücksichtigt bleiben. Dazu gehört auch die Verquickung persönlicher mit kommerziellen Interessen. Als Beispiel nennt Bleuler die Verordnung teurer und fragwürdiger Medikamente $^{21}$. Eine grosse «Arzneimittelindustrie» lebe vom «autistischen Denken der Patienten und der Ärzte» ${ }^{22}$. Patient, Arzt, Wissenschaft und Gesellschaft erscheinen in Bleulers Darstellung als eine Einheit, die sich durch wechselseitige, eben nicht eindimensionale Abhängigkeiten auszeichnet. Im autistischen Denken sind nicht nur die Ärzte, sondern auch die Patienten, deren Verwandte, kurz all diejenigen befangen, die mit einer Krankheitssituation in Berührung kommen.

Die bisher genannten Aspekte «autistischer» Verhaltensweisen meinen in erster Linie die Praxis der Medizin. Genauso vom «Autismus» betroffen ist jedoch ihr theoretischer Konsens, so, wenn mit als bekannt vorausgesetzten Begriffen gearbeitet werde, die dabei aber nur «im vulgärsten Sinne» verwendet würden, ohne dass sie überhaupt wissenschaftlich definiert $\operatorname{seien}^{23}$. Bei der Erstellung von wissenschaftlichen Publikationen stehe der «Grundfehler» der «affektiven Einstellung zu einem zu erwartenden Resultat» ganz im Vordergrund. Fehlerhafte Interpretationen von Forschungsergebnissen auf dem Boden autistischen Wunschdenkens seien hier

20 Op. cit., S. 17. «Das auffälligste im Formellen des schizophrenen Gedankenablaufs sind die Sperrungen. Die Assoziationstätigkeit steht manchmal plötzlich ganz still; wenn sie wieder einsetzt, tauchen meist Ideen auf, die mit den vorhergehenden in keinem oder nur ungenügendem Zusammenhang stehen» (Bleuler 1911, S. 26).

21 Bleuler 1919, S. 15.

22 Op. cit., S. 14.

23 Op. cit., S. 59f. Bleuler nennt hier den Begriff der Krankheit als Beispiel. 
vorprogrammiert. Auch hier, in bezug auf die Theorie, erhebt Bleuler den Vorwurf, dass man Thesen «viel zu wenig an der Wirklichkeit» nachprüfe ${ }^{24}$.

Bleulers oft derbe Wortwahl hat Methode. Der Stil markiert in seiner betonten Bodennähe den Gegenpol zur «akademischen Medizin». Eugen Bleuler und ebenso später sein Sohn Manfred Bleuler (1903-1994) wurden nie müde, ihre Herkunft aus bäuerlichem Zollikoner Geschlecht zu betonen, auch zu stilisieren. Das bäuerliche Denken war das gerade Denken, das sich nur nach Erfahrungen und Beobachtungen, nicht aber nach Moden richtet; es war ein Denken, das vor allem anderen patientennah und praktisch zu sein hatte ${ }^{25}$. Für Eugen Bleuler sind es «Fehler der Denkmethodik», die für den Zustand der Medizin verantwortlich $\operatorname{sind}^{26}$. Es fehlten die «realen Unterlagen» des Denkens: das sind für ihn in erster Linie die objektive Beobachtung und die «Introspektion». Nur in der bewussten Trennung von objektivem Schauen und subjektivem Folgern können grundsätzliche Fehler in der Denkmethodik vermieden werden.

Bleulers Kritik ist Positivismuskritik - oder besser, Kritik an der Interpretation des Positivismus. Vordergründig beanstandet er die mangelhafte Umsetzung theoretischer Erkenntnisse. Dahinter steht aber die Warnung vor einer Überbewertung dieser Erkenntnisse im Hinblick auf einen absoluten Wahrheitsanspruch. Ersteres ist Kritik am ärztlichen Stand, bitter und herausfordernd zwar, aber durch geeignete Verhaltensmassregeln prinzipiell zu lösen. Nicht so leicht fassbar ist die zweite Ebene von Bleulers Kritik am «autistisch-undisziplinierten Denken in der Medizin»: hier offenbart sich eine grundsätzliche Skepsis gegenüber der Möglichkeit des Erkennens mit den Mitteln, die die positivistische Wissenschaft auszeichnen. Das beim Autismus gestörte Verhältnis von Innen- und Aussenwahrnehmung findet Bleuler im Wissenschaftsverständnis moderner Medizin wieder. Psychiatrie und Psychologie sollten dieses Verhältnis in ein neues Gleichgewicht bringen, indem sie den notwendig gewordenen Einblick in die Arbeit des menschlichen Geistes anbieten könnten.

\section{Das Dilemma des modernen Arztes: Arzt und Psyche}

Das Dilemma des Mediziners ergibt sich für Bleuler aus dem besonderen Spannungsverhältnis zwischen Erwartung und Erfüllung, das die Medizin

24 Op. cit., S. 196.

25 Vgl. Bleuler, Manfred: Zur Entstehung und Bedeutung von Eugen Bleulers Werk: «Dementia praecox oder Gruppe der Schizophrenien». In: Praxis 77 (1988), S. 1322-1326.

26 Bleuler 1919, S. 23. 
von allen anderen Naturwissenschaften unterscheide. Die Erwartung liegt hier nicht nur beim Arzt, der wünscht, seinem Patienten zu helfen, sondern sie kommt umgekehrt auch vom Patienten auf ihn zurück. Die Erfüllung, ein geheilter Patient, steht als absolute Forderung im Raum. In ihr manifestiert sich das transzendente Moment des Arztberufs als Heilserwartung von seiten des Patienten, die der Arzt zu erfüllen hat und selber auch immer wieder erfüllen will. Da dieses aber in den meisten Fällen nicht möglich ist, oft nur eine Herauszögerung des Unausweichlichen bedeutet, befindet sich der Arzt in einer eigentümlichen Spaltungssituation ${ }^{27}$. Um hier einen Ausweg zu finden, greift er - analog zur Ätiologie autistischen Denkens bei der Schizophrenie - aufs Affektive zurück. Das vermag ihn und oft auch seinen Patienten zwar zu erleichtern, verstellt ihm aber gleichzeitig den Blick für die Realität. Diese Realität wiederum ist ebenfalls mehrdimensional und kann nur erfasst werden, wenn sie als ein vom Subjekt abhängiger Prozess begriffen wird. Objektivität kann für Bleuler nur unter Einbeziehung der Subjektivität entstehen.

Ebenso muss die Wissenschaft an die Probleme herangehen. Die Erkenntnistheorie der Medizin muss diesen Aspekt der psychischen Interaktion berücksichtigen ${ }^{28}$. Gelingt das nicht, kommt die Objektivität als Gradmesser für Wissenschaftlichkeit und Nachprüfbarkeit zu kurz: der Wissenschaftler im Arzt sinkt auf die Stufe kindlichen Wünschens zurück ${ }^{29}$. Die Wissenschaftlichkeit der Medizin im herkömmlichen Sinne sei damit nicht mehr gewährleitet, sie sei so auch gar nicht möglich. Bleuler gibt der Medizin eine Sonderstellung im Reigen der Wissenschaften, deren Begründung im Subjektiven liegt.

Eines der zentralen Anliegen der Medizin, fest verankert mit ihrem Selbstverständnis spätestens seit Hippokrates, ist das Spenden von Trost und Zuspruch. Der Akt der Tröstung des Patienten durch den Arzt, gerade da, wo die Wissenschaft am Ende ist, ist zugleich die intimste Verschränkung dieses einzigartigen Subjekt-Objekt-Verhältnisses. Die persönliche Betroffenheit ist dabei vielschichtig, denn der Arzt wird nicht nur von den Wünschen seines leidenden Patienten gelenkt, er ist selbst ja auch ein potentieller Patient und nicht frei von den Ängsten seines Gegenübers. Bleuler spricht in diesem Zusammenhang von den «instinktiven Bedürfnis-

27 Zum Beispiel op. cit., S. 2.

28 Vgl. Bleuler, Eugen: Psychophysischer Parallelismus und ein bisschen andere Erkenntnistheorie. In: Zeitschrift für Psychologie 41 (1905).

29 Das Wünschen wie beim Kind, das das Aussprechen eines Wunsches bereits als dessen Erfüllung betrachten kann. Bei dieser Art von Wünschen verschwimmt die Grenze zwischen Subjekt und Aussenwelt. 
sen», die das «medizinische Denken auf Abwege führen», weil die Medizin «unser Wohl und Wehe» direkt berühre ${ }^{30}$. Bleuler kritisiert, dass sich die Mediziner dieser Interaktionen gar nicht «bewusst» seien. Er macht hier nichts anderes als den Versuch, die Situation der Medizin anhand der agierenden Partner zu analysieren: Arzt und Patient werden von ihm im Hinblick auf ihre Wechselbeziehung betrachtet.

In der grossen Bedeutung, die Bleuler dem Begriff «bewusst» ${ }^{31}$ in seinem Werk einräumt, zeigt sich seine enge Verbindung zur Psychoanalyse. Letztlich fordert er mit seiner kleinen Streitschrift die Selbstanalyse der Ärzteschaft im Hinblick auf die Verbesserung ihrer medizinischen Leistung. Es wird recht deutlich, dass sich Bleuler viel vom Prozess der Bewusstmachung verspricht, wie er zum Beispiel mit der Hypnose erreicht werden könne. Bleulers Beschäftigung mit der Freudschen Analyse reichte bis in die späten neunziger Jahre des letzten Jahrhunderts zurück. Der starke Einfluss von Sigmund Freud (1856-1939) auf Bleulers psychiatrische Konzepte ist bekannt ${ }^{32}$; aber auch Bleulers Denken über die wissenschaftliche Konzeption der Medizin wurde vom Wiener Kollegen entscheidend geprägt ${ }^{33}$. Ein zentrales Element aus dieser Schulung war die positive Grundeinstellung gegenüber der Hypnose. Bleuler, der hypnotische Techniken in zahlreichen Varianten zumeist in Selbsterfahrung ausprobiert hatte, fand die Hypnose besonders für die Therapie neurotischer Störungen seiner Patienten geeignet ${ }^{34}$. Freud hatte seine Betrachtungen über die Anwendung der Hypnose mit dem mehrdeutigen Zusatz versehen, dass «in naturwissenschaftlichen Dingen stets nur die Erfahrung und nie die Autorität ohne Erfahrung die endgültige Entscheidung über Annahme und Verwerfung herbeiführt $»^{35}$. Wie Freud wollte Bleuler erreichen, dass die Hypnose mit den gleichen Mitteln wissenschaftlichen Objektivierens geprüft werde wie andere Methoden auch. Eine Forderung, die an der von ihm bitter konstatierten Abwehrhaltung eines

30 Bleuler 1919, S. 4.

31 Bleuler betont die Trennung von «Bewusstsein» und «Introspektion»: «Für diejenigen, die hier erkenntnistheoretische Erwägungen hineintragen möchten, sei bemerkt, dass die Introspektion, soweit sie hier in Betracht kommt, nicht identisch ist mit dem Bewusstsein, noch eine Folge der bewussten Qualität, sondern eine Parallelerscheinung derselben» (Bleuler 1919, S. 158). Vgl. dazu Bleuler 1916, S. $2 \mathrm{ff}$.

32 Im Vorwort der «Dementia praecox» von 1911 schrieb er, dass er hier ja eigentlich nichts anderes gemacht habe, als die Freudschen Theorien für die Praxis auszuarbeiten.

33 Bleuler 1919, S. 52 ff.

34 Bleuler hatte schon in den 80er Jahren des 19. Jahrhunderts begonnen, mit Hypnose zu experimentieren, und seine Erfahrungen publiziert. Vgl. Bleuler, Eugen: Der Hypnotismus. In: Münchner Medizinische Wochenschrift 37 (1887); ders.: Zur Psychologie der Hypnose. In: Münchner Medizinische Wochenschrift 5 (1889).

35 Freud zitiert nach Gay, Peter: Freud. Frankfurt a. M., Fischer, 1995, S. 65. 
Grossteils seiner Kollegen scheiterte ${ }^{36}$. Die Furcht vor der Anwendung der Hypnose war für Bleuler Resultat einer allgemeinen «Psychophobie des modernen Arztes», der alles, was nur im entferntesten mit der Psyche zu tun haben könnte, als unwissenschaftlich ablehne ${ }^{37}$. Die Ablehnung dieser Methode beruhe nicht auf empirischen Tatsachen, sondern habe ihre tiefere Ursache in der Angst der Ärzte vor der Aufdeckung ihrer eigenen Psyche und sei somit «autistisch»-irrationaler Natur ${ }^{38}$. Bleulers Kampf um die Hypnose als Technik der Diagnose und Therapie verschränkt sich hier mit seiner Hoffnung auf den Durchbruch, den die Kenntnis des Unbewussten für das ärztliche Denken selbst haben könnte.

\section{Die Reaktion}

Bleuler war es klar, dass diese Schrift nicht nur auf Unverständnis, sondern sogar auf Empörung treffen würde ${ }^{39}$. Im Vorwort zur zweiten Auflage erwähnt er «Angriffe», die ihm immerhin gezeigt hätten, dass er «nicht auf falschem Wege» $\mathrm{sei}^{40}$. Sein Sohn Manfred Bleuler (1903-1994) berichtete später, die Rezensenten hätten das Buch in Stücke gerissen und dem Autor vorgeworfen, Würde und Ethik der Medizin untergraben zu haben ${ }^{41}$. Um 1920 soll die Diskussion um Bleulers Buch in Zürich einen Höhepunkt erreicht haben ${ }^{42}$. Der Tenor der Vorwürfe bezog sich auf die Kritik an der Medizin als Profession. Praktiker wiesen auf die Undurchführbarkeit bestimmter, von ihnen prinzipiell als richtig anerkannter neuer Methoden $\mathrm{hin}^{43}$. Zustimmung kam von Nervenärzten, die die Bedeutung «der psychischen Seite der Krankheiten» hervorhoben. Missverstanden wurde Bleulers Ansatz, wenn betont wurde, man käme eben bei den «Psychoneurosen» mit «exakter Naturwissenschaft» nicht weiter und müsse sich der «Psychologie» bedienen. Genau diese Trennung zwischen einer «naturwissenschaftlichen Seite» und der psychologischen Betrachtungsweise wollte Bleuler vermei-

36 Bleuler 1919, S. 49.

37 Op. cit., S. $51 \mathrm{ff}$.

38 Op. cit., S. 50.

39 Op. cit., S. IV.

40 Bleuler, Eugen: Das autistisch-undisziplinierte Denken in der Medizin und seine Überwindung. Zweite, verbesserte Auflage. Berlin, Springer, 1921, S. VI.

41 Bleuler, Manfred: «On Eugen Bleuler and how his book on «Autistic Thinking〉 came to be written». Vorwort zur 1. amerikanischen Ausgabe. Darien, Hafner, 1970, S. XVI.

42 Harms, Ernest: «Eugen Bleuler and America». Einleitung zur 1. amerikanischen Ausgabe, S. VII.

43 Schmitt in: Medizinisches Korrespondenzblatt für Württemberg 99 (1929), S. 470. 
den ${ }^{44}$. Bleulers erkenntniskritischer Ausgangspunkt wurde nur zum Teil wahrgenommen ${ }^{45}$.

«Man wirft mir vor, ich übersähe, dass die Ärzte oft zwar wissenschaftlich denken, aber trotzdem notgedrungen autistisch handeln müssen, was ich doch selber gesagt habe; oder ähnlich, ich vergesse, dass die Medizin nicht blosse Wissenschaft, sondern auch eine Kunst sei.» ${ }^{46}$

Sorgfältige Antwort gab er in der zweiten Auflage nur einem seiner Kritiker ${ }^{47}$ : Constantin von Monakow (1853-1930) war, wie zu seiner Zeit nicht unüblich, Psychiater und Neurologe in einer Person ${ }^{48}$. Von ihm stammt eine differenzierte Rezension, die uns eine Vorstellung von dem grossen Bezugsrahmen gibt, in den Bleuler seine Analyse des medizinischen Denkens gestellt hatte. Monakow stand in langjährigem persönlichen und beruflichen Kontakt zu Eugen Bleuler, was sich in auffälligen Parallelen ihres wissenschaftlichen Denkens niedergeschlagen hat ${ }^{49}$. In seiner Kritik geht es Monakow besonders um die Aspekte in Bleulers Schrift, die das Verhältnis von Psychologie und Biologie beleuchten, und damit um eine Thematik, die ihn in diesen Jahren selbst beschäftigte ${ }^{50}$. Bleulers oben zitierte Anspielung auf den Einwand, dass die Medizin eben keine exakte Wissenschaft, sondern vielmehr eine Kunst sei, scheint auf eine bestimmte Passage in Monakows Rezension abzuzielen:

«Aber die Medizin ist auch streng genommen keine Wissenschaft (ebensowenig wie die Staatswissenschaft), wenn sie aus anderen, experimentellen Wissenschaften (Chemie, Phy-

44 Stockmayer, W.: Bemerkungen zu Schmitts Ausführungen über Bleulers Autistisches Denken in der Medizin. Op. cit., S. 521. Vgl. auch die Neubesprechung durch Gunther Kruse, in: Psychiatrische Praxis 23 (1996), S. 255-256.

45 Vgl. Gärtner, Johannes: Die Bedeutung Eugen Bleulers für die Entwicklung in der allgemeinpraktischen Medizin. In: Landarzt 41 (1965), S. 187-191. In dieser Arbeit wird der Gedanke Bleulers vom «kritischen Messen an der Wirklichkeit» als besondere«Eigentümlichkeit der Allgemeinmedizin» herausgestellt und dieser ein eigener Erkenntniswert zugedacht.

46 Bleuler 1921, S. V.

47 Op. cit., S. 71. Er nennt Monakow nicht namentlich.

48 Siehe dazu Jagella, Caroline; Isler, Hansruedi; Hess, Klaus: Constantin von Monakow (1853-1930). Hirnforscher - Neurologe - Psychiater - Denker. In: Schweizer Archiv für Neurologie und Psychiatrie 145, Supplementum I/1994.

49 Das Verhältnis zwischen Monakow und Bleuler war freundschaftlich, wenn auch nicht ohne Ambivalenzen. Die jahrelange Zusammenarbeit zwischen Bleuler als Direktor des Burghölzli (1898-1927) und Monakow als Direktor des Zürcher hirnanatomischen Instituts und der Nervenpoliklinik (1894-1927) fand vor allem auf dem Gebiet der klinischen Medizin statt. Man schickte sich Patienten zum Konsilium oder bat um Beurteilung hirnpathologischer Befunde. Im sogenannten «Monakowschen Kränzchen» kam man während einiger Jahre regelmässig zur interdisziplinären Patientenvorstellung zusammen.

50 Monakow, Constantin von: Kritische Besprechung nebst eigenen psycholog.-biol. Betrachtungen von E. Bleuler: Das autistisch-undisziplinierte Denken in der Medizin und seine Überwindung. In: Schweizer Archiv für Neurologie und Psychiatrie 7 (1920), S. 167-186; vgl. Seite 169.

51 Op. cit., S. 168. 
siologie, Biologie) nach Möglichkeit zu schöpfen sucht, sondern eine Kunst, und der gute Arzt ist mehr Künstler als Wissenschafter. Bei jeder Kunst spielen aber die Welt der Gefühle und die Intuition (...) eine mindestens ebenso grosse Rolle wie die Welt exakter Orientierung. $»^{51}$

Monakow wirft Bleuler vor, dass dieser «die menschliche und die wissenschaftliche Seite nicht genügend auseinanderhält und bald als Mensch (in fast vulgärer Sprache) und bald als Wissenschafter (in sorgfältig gegliederter und überlegter Form) seine Gedanken» niederlege. Bleulers «autistisch» sei eigentlich nicht «neu» und mit Synonymen wie «Vorurteil, vorgefasste Meinung, persönliches, subjektives Denken, Voreingenommenheit, Befangenheit, Parteilichkeit» gleichzusetzen. Ausserdem habe er «autistisch» nicht klar definiert; auf das Denken sei dieser Begriff schon gar nicht anwendbar, weil es hier niemals eine prinzipielle, sondern nur eine graduelle Unterscheidung geben könne. Die Differenzierung in «autistisch-undiszipliniert» und in logisch-«diszipliniert» sei einfach nicht haltbar. Monakow fordert von Bleuler eine «biologische Begründung» seiner Thesen. Er stellt lakonisch fest, dass «ein gewisses Mass an Autismus» bei «jeder Beurteilung menschlicher Verhältnisse» mitarbeite ${ }^{52}$. Ihm kommt es ganz besonders auf die Feststellung an, dass der Arzt immer nur als «biologisches» Wesen urteile und seine Urteile deshalb auch immer nur Teil des biologischen Systems sein könnten. Wirkliches Verständnis dieser seelisch-geistigen Vorgänge ist für Monakow nur auf der Basis einer differenzierten Kenntnis der biologischen Entwicklung der Denkprozesse möglich. Eine «psychologische Betrachtungsweise, die aus dem Leben geschöpft wurde», könne nie «naturwissenschaftlich» sein in dem Masse, wie es das Studium der Morphologie und ihrer individuellen Entwicklungsgeschichte ermögliche und verlange ${ }^{53}$. Letztlich sei Bleulers Schrift selbst wiederum ein Zeugnis autistischen Denkens. Denn in der Nichtberücksichtigung biologischer, also exaktnaturwissenschaftlicher Grundlagen des Denkens habe er den Boden der Tatsachen selbst verlassen ${ }^{54}$. Auch Bleulers Bewertung der Hypnose und seine stürmische Verurteilung der Kollegen, die diese als Mittel der (Selbst)Erkenntnis ablehnten, kann Monakow nicht teilen: die Lehre von Hypnose und Suggestion eigne sich nicht für eine breite Anwendung, zudem hafte ihr ein «gewisses 〈unreelles»» Moment an, und sie könne nicht von jedem Arzt ausgeführt werden ${ }^{55}$. 
Monakows Kritik an Bleulers grossangelegtem Sittenbild vom «autistischundisziplinierten Denken» in der Medizin hat prinzipiellen Charakter. Im Mittelpunkt seiner umfangreichen Diskussion der Bleulerschen Thesen steht der Vorwurf der unklaren Begriffsabgrenzung und damit eines der quälendsten Probleme neurowissenschaftlicher Forschung, da es um die Grenze zwischen naturwissenschaftlichen und philosophischen, allgemein-weltanschaulichen Urteilen geht. Bleuler unterscheide nicht klar zwischen seinen Urteilen als Mensch und denen, die er als Wissenschaftler fälle ${ }^{56}$. Eine «vulgäre» ${ }^{57}$ Psychologie könne nicht auf komplexe biologische Zusammenhänge übertragen werden, und die Äquivalente des Denkens und Fühlens seien im Menschen nie zu trennen. Eine klare Trennung und Definition der Begriffe und ihrer Wertung sei nur im Sinne der wissenschaftlichen Biologie zu erreichen.

Inwieweit Monakow Bleuler bewusst missverstand, um vor diesem Hintergrund seine eigene Vorstellung einer «modernen biologischen Psychologie und Psychotherapie» pointierter darlegen zu können, muss dahingestellt bleiben ${ }^{58}$. Die «Psychobiologie» war zum zentralen Anliegen in Monakows Spätwerk geworden ${ }^{59}$. Auch Bleulers Altersarbeiten standen unter der Ägide psychobiologischer Überlegungen ${ }^{60}$. Monakow und Bleuler teilten im Alter die Vision von einer neuen «biologischen Psychiatrie» oder «Neurobiologie» oder «biologischen Psychologie», in der Psyche und Leib endlich naturwissenschaftlich vollkommen ineinander aufgehen könnten $^{61}$. Ihre Anschauungen trennten Welten, wenn es um die Grundlage dieser Zusammenschau von Neurologie und Psychiatrie ging ${ }^{62}$. Der Diskurs über das «autistisch-undisziplinierte Denken in der Medizin» wird an diesem

56 Op. cit., S. $182 \mathrm{f}$.

57 Vgl. dazu Monakow, Constantin von: Fünfzig Jahre Neurologie. Zürich, Orell Füssli, 1924, S. 40 .

58 Im Grunde sind sich beide in ihrer Intention sehr nahe, wenn sie eine klare Begriffsabgrenzung fordern; Monakow scheint die Differenzierung, die Bleuler zwischen den Bereichen des ärztlichen Handelns und des ärztlichen Denkens macht, nicht wahrzunehmen.

59 Siehe zum Beispiel Monakows Arbeiten «Psychiatrie und Biologie» (1918/1919); «Versuch einer Biologie der Instinktwelt» (1921/1922); "Grundlagen der biologischen Psychiatrie» (1925); «Die Syneidesis, das biologische Gewissen» (1927); «Introduction Biologique à l'étude de la Neurologie et de la Psychopathologie» (1928); «Psychologie, Biologie und Neurose» (1930).

60 Siehe zum Beispiel Bleulers Arbeiten «Ein Stück Biopsychologie» (1929); «Mechanismus Vitalismus - Mnemismus» (1931); «Die Psyche als Lebensfunktion» (1932); «Mnemistische Biologie und Psychologie» (1936).

61 Beide wollten psychologischen Phänomenen den gleichen Anspruch auf «Wissenschaftlichkeit» einräumen wie den physiologischen oder biologischen Grössen.

62 Der Konflikt in Schlagworten: Morphologie und Biologie auch für Psyche und Moral als unabdingbare Grundlage neurologischen und psychiatrischen Denkens auf der einen Seite - Erkenntnisgewinn durch Introspektion und Verlassen primär physiologischer Terminologie auf der anderen. 
Punkt zum Diskurs um die Zukunft von Neurologie und Psychiatrie ${ }^{63}$. Wenn Monakow zusammenfasst, die von Bleuler «angewandte Argumentation» erinnere «mehr an Plaidoyers «überscharfsinniger» Anwälte, aber nicht an math.-physikal. Denkmethoden», so kennzeichnet und polarisiert er den Konflikt, an dem letztlich die Gemeinschaft von Neurologie und Psychiatrie auseinanderbrechen sollte ${ }^{64}$.

An Monakows Antwort wird aber auch deutlich, welch exemplarische Bedeutung der Frage nach dem Verhältnis von Biologie und Psyche zukam. Durch die Psychoanalyse wurde die wissenschaftliche Methodik der modernen Medizin in Frage gestellt ${ }^{65}$. Neurologie und Psychiatrie trugen hierüber einen Konflikt aus, der als richtungsweisend empfunden wurde, weil es hier um mehr ging als um Abgrenzungsversuche zweier Spezialdisziplinen. Der holländische Neurologe Cornelis Winkler (1855-1941) schrieb im November 1920 an seinen Freund Monakow:

«Ich habe mich sehr amüsiert mit Ihrer Kritik über das Büchlein von Prof. Bleuler. Es ist vielleicht am besten es auf diese[r] Weise zu behandeln, aber unerhört bleiben doch solche Anfälle, auf unsere Kunst.» ${ }^{66}$

Winkler formuliert Bedenken gegen die «Methodik der Untersuchung»: die «Psycho-analyse Freud's» sei «keine Wahrnehmung mehr». Das Thema wurde in einem späteren Schreiben nochmals aufgenommen; wieder nahm Winkler die Rezension Monakows zum Anlass:

«Ich verstehe, dass Prof. Bleuler sehr gereizt ist. Aber verdienen diese Leute [es] besser? In der Schweiz, wie hier in Holland, überall ist es das Selbe. Sie wollen die Psychiatrie als ein Fach ausgeben, das an der Spitze der Wissenschaft steht. Dafür sind physiologische und anatomische Kenntnisse unnötig. Stattdessen macht man alles und noch etwas, nennt es Psychologie, will die ganze Medicin von philosophischem Denken durchdringen (...) Nein. Ihre Kritik über das autistische Denken ist ausserordentlich milde. M. E. ist alles darin unerlaubt.» ${ }^{67}$

63 Monakow deutet diese Kluft an, wenn er von Bleulers «psychiatrischer Erfahrung» schreibt und kurz danach seine «eigene neurologische Erfahrung» hervorstreicht (Monakow 1920, S. 185f.).

64 Op. cit., S. 184.

65 Monakow und Bleuler waren 1904 Gründungsmitglieder des «psychiatrisch-neurologischen Vereins» in Zürich, der später wegen der grundsätzlichen Verschiedenheit der Auffassungen über die Psychoanalyse eine Aufspaltung in die «Freudsche Vereinigung» und in den neurologischen Verein erlebte; vgl. Jagella et al. (oben Anm. 48), S. 44ff.

66 Unveröffentlichtes Schreiben vom 9.11.1920. Briefsammlung des Medizinhistorischen Instituts und Museums der Universität Zürich. Winkler gilt als Begründer der holländischen Neurologie. Seine Arbeit wurde von Monakow beeinflusst. Vgl. dazu Winkler, Cornelis: Herinneringen. Arnhem, Van Loghum, 1947; ders.: Die Bedeutung der Arbeit Constantin von Monakow's für die Wissenschaft. Schweizer Archiv für Neurologie und Psychiatrie 13 (1923); Lhermitte, Jean; Mourgue, Raoul: La vie et l'œuvre de Cornélis Winkler. In: L'Encephale 4 (1946/47).

67 Unveröffentlichtes Schreiben vom 27.12.1920. Briefsammlung des Medizinhistorischen Instituts und Museums der Universität Zürich. 
Winkler hat Bleulers Konzept einer naturwissenschaftlichen Psychologie und psychologischen Naturwissenschaft zwar missverstanden, seinen epistemologischen Ansatz aber wahrgenommen.

\section{Das Dilemma der modernen Medizin: Arzt und Gesellschaft}

Vielleicht das Eigenartigste an Bleulers Reflektionen über das «autistischundisziplinierte Denken in der Medizin» ist der Versuch, eine tief empfundene Krise der Medizin mit einem Begriff aus der Medizin selbst zu erfassen. Bleuler skizziert hier eine Art Psychogramm ärztlicher Befindlichkeit, womit er die Medizin seiner Zeit als heilungsbedürftig hinstellt. Indem er die Ärzte auf die Stufe von Patienten stellt, macht er sie zum nun plötzlich mitagierenden und mitreagierenden Teil eines ganzen Bezugssystems. Der ärztlichen Psyche, ihrer Subjektivität, wird in diesem System zentrale Bedeutung zugemessen, und zwar sowohl in bezug auf das therapeutische Verhältnis zum Patienten als auch in bezug auf die wissenschaftliche Arbeit des Forschers. Dadurch wiederum entsteht der Eindruck der Relativität menschlicher Willens- oder Geisteshaltungen - eine Vorstellung, die besonders für die Naturwissenschaften weittragende Konsequenzen in sich birgt ${ }^{68}$. So wie Bleuler ohne grosse Hemmungen psychopathologische Phänomene in gradueller Abstufung «auch bei Gesunden» findet, so ist auch die Mitbeeinflussung der Forschung durch affektive Bedürfnisse für ihn etwas Selbstverständliches. Die Zerlegung einer «natürliche[n] Einheit» ${ }^{69}$, wie sie Bleuler im menschlichen Denken annimmt, kann auch in der Wissenschaft nur kontraproduktiv $\operatorname{sein}^{70}$. Krankheit und Gesundheit sind nur relative Zustände, ebenso kann die Begriffsbildung nur dieses relative Verhältnis spiegeln. Die Vorstellung, man müsse sich nur auf Statistiken berufen und schon halte man den Garanten für naturwissenschaftliche Objektivität in den Händen, führt er ad absurdum ${ }^{71}$.

68 «Die an anderen Orten so lebhaft ventilierte Frage, ob es einen sfreien Willen sebe in dem Sinne, dass ohne Ursache die Entscheidung getroffen werden könne, besteht für die Naturwissenschaft nicht. Wir sehen, dass die Handlungen der belebten Geschöpfe durch die innere Organisation und die darauf einwirkenden äusseren Einflüsse genau so determiniert sind wie irgendein anderes Geschehen» (Bleuler 1916, S. 36).

69 Op. cit., S. 225. Auch in der Natur gebe es solche Trennlinien nicht.

70 Die affektive Einstellung zu einem «zu erwartenden Resultat» sei zwar ein grosses Problem in der naturwissenschaftlichen Forschung, habe aber insofern ihr Gutes, «als eben nur das affektbetonte Streben über maximale Kräfte verfügen kann» (Bleuler 1919, S. 196); vgl. Bleuler 1916, S. 16.

71 Bleuler 1919, S. $108 f$. 
Bleuler greift mit seiner Streitschrift, so burschikos sie daherkommen mag, die uralte Diskussion um ratio et experientia in der Medizin wieder auf. Es ist die Diskussion um die Frage, ob und wie zwischen Spekulation und Empirie, zwischen subjektiver und objektiver Erfahrung der Erscheinungen, sicher unterschieden werden kann. Wenn Bleuler diese Frage, wie auch ihre Antwort, relativiert, so spricht er für das 20. Jahrhundert ${ }^{72}$.

Eugen Bleulers Diagnose «autistisch-undisziplinierten Denkens in der Medizin» beruht auf seiner Arbeit als Psychiater. Er übernimmt dabei nicht nur die Terminologie seiner psychiatrischen Konzepte: es ist die Analyse von Denken ${ }^{73}$, Bewusstein ${ }^{74}$ und Unbewusstem ${ }^{75}$ aus der "Dementia praecox» und dem "Lehrbuch der Psychiatrie», die hier nun zur Analyse ärztlichen Denkens und Handelns wird. Das ärztliche Urteil, bislang in den Bereichen von Kunst und Intuition bewahrend angesiedelt, kann sich - mit Bleuler gedacht - nach diesen Vorgaben einer gleichwiegenden Analyse nicht mehr verschliessen ${ }^{76}$. Die Spaltung der «wichtigsten psychischen Funktionen», die Bleuler 1911 als Kennzeichen der Schizophrenie definiert hatte ${ }^{77}$, wird 1919 auf das Denken und Handeln der Medizin selbst bezogen.

72 «Die psychische Reihe hat also absolute Realität, aber nur für die betreffende Psyche; diese Realität ist folglich eine subjektive. Für die Existenz der Aussenwelt aber gibt es keine Beweise. Dass der gesehene Tisch existiert, ist nur eine Annahme, wenn auch eine praktisch notwendige (...). Die Realität der physischen Welt ist also eine unsichere, relative, aber dafür eine objektiv demonstrierbare» (Bleuler 1916, S. 5).

73 Bleuler sieht das Denken in enger Beziehung zu den Assoziationen, die wiederum sehr von individuellen Faktoren wie zum Beispiel Erinnerungen abhängig seien; vgl. Bleuler 1916, S. 14.

74 «Wir bezeichnen mit diesem Worte ein undefinierbares Etwas, eine Qualität psychischer Vorgänge. Es ist diejenige, die uns am deutlichsten vom Automaten unterscheidet» (op. cit., S. 2). Bleuler gibt hier den kleinsten gemeinsamen Nenner der Vorstellungen vom Wesen des Bewusstseins. Er selbst lässt sich auf keine eigentliche Neudefinition des Bewusstseinsbegriffs ein, sondern zeigt vielmehr die Schwachpunkte gängiger - medizinischer und philosophischer - Vorstellungen auf. Er differenziert und grenzt ab, ohne selbst zu definieren.

75 «Alles, was in unserem Bewusstsein vorkommt, kann also auch unbewusst ablaufen. In diesem Sinne gibt es unbewusste psychische Vorgänge. Die psychische Determination ist ferner nur dann eine vollständige, wenn diese unbewussten Vorgänge in die Kausalkette mit eingeschlossen werden.» Dann begründet er: «Es ist für die Wissenschaft notwendig, dieselben zu den psychischen zu rechnen, nicht nur, weil sie in allem, abgesehen von dem Mangel der bewussten Qualität, der bewussten gleichkommen, sondern vor allem deshalb, weil die Psychologie und namentlich die Psychopathologie nur dann eine erklärende Wissenschaft sein kann, wenn man so wichtige Ursachen der Phänomene mitberücksichtigt» (op. cit., S. 6).

76 «Das Urteilen besteht in einem Wiederholen von Zusammenhängen, die die Erfahrung gegeben hat. 〈Der Schnee ist weiss〉, 〈Kant war ein grosser Mann〉, sind der Ausdruck von direkten und indirekten Erfahrungen. Es ist aber wichtig, zu beachten, dass das Wort 〈Urteil〉 zwei Dinge bedeutet: In der Logik ist ein Urteil «die Form, in der Erkenntnisse gedacht und ausgesprochen werden〉. Wenn man aber in der Psychiatrie von «Urteilsfähigkeit> spricht, so meint man die Fähigkeit, Urteile zu gewinnen, also das Vermögen, richtige Schlüsse aus Erfahrungsmaterial zu ziehen» (op. cit., S. 14f.).

77 Bleuler 1911, S. 5. 
Zwischen der Veröffentlichung der «Dementia praceox» und dem «autistisch-undisziplinierten Denken in der Medizin» liegen acht Jahre, die die Jahre des Ersten Weltkriegs umfassen. Die Herausgabe einer Arbeit, in der es um die Unwägbarkeiten und Unberechenbarkeiten des Subjektiven ging, just im Jahr 1919 war kein Zufall. Es waren drei Ereignisse, die, jedes für sich, durch ihre Wirkung und ihre immanente Aussage jedoch zueinander in Beziehung stehend, das Denken dieser Jahre prägten: der Erste Weltkrieg, die Influenzaepidemie und die Anerkennung von Einsteins Relativitätstheorie. Alle drei Ereignisse finden einen Widerhall in Bleulers Arbeit, ausgesprochen und, vielleicht noch schwerwiegender, unausgesprochen. Vor allem anderen war der Erste Weltkrieg ein Trauma, ein Einschnitt im Bewusstsein Bleulers wie im Bewusstsein der Bevölkerung Europas, mit tiefst- und weitestgehenden Folgen. Von der Krise der Gesellschaft waren die Wissenschaften nicht ausgeschlossen, am allerwenigsten vielleicht die Medizin. In der Medizin zeigten - und zeigen - sich eklatante Werteverluste am deutlichsten, ihr Selbstverständnis ist von jeher aufs engste mit den gesellschaftlich-politischen Verhältnissen verknüpft. Das Trauma des Ersten Weltkriegs traf den einzelnen Forscher nicht nur in materiell-persönlicher Hinsicht, es hatte direkte Auswirkungen auf sein Denken als Wissenschaftler. Die Relativierung der Wertvorstellungen, wie sie der Krieg mit sich brachte, hielt in die Wertung wissenschaftlicher Aussagen und Erkenntnisse Einzug. Freud registrierte 1915, auch für sich selbst:

«Von dem Wirbel der Kriegszeit gepackt, einseitig unterrichtet, ohne Distanz von den grossen Veränderungen, die sich bereits vollzogen haben oder zu vollziehen beginnen, und ohne Witterung der sich gestaltenden Zukunft, werden wir selbst irre an der Bedeutung der Eindrücke, die sich uns aufdrängen, und an dem Werte der Urteile, die wir bilden.» ${ }^{78}$

Der «grosse Krieg», wie ihn Bleuler 1919 immer wieder zitiert, war das Mass, an dem altbekannte und nie in Frage gestellte Diagnosen und Therapien nun gemessen werden mussten. Die vielen Kriegsverletzten und Seuchenopfer, an denen die Hilflosigkeit oder aber auch die Überflüssigkeit der Medizin in den Kriegsjahren offenbar wurde, forderten ein Umdenken. «Der Krieg mit seinen tausenden von Neurosen der positiven und negativen Begehrung ${ }^{79}$ hatte den Psychiater und den Menschen mit neuen Dimensionen menschlichen Verhaltens in Berührung gebracht, die es dem Wissenschaftler unmöglich machten, wie bisher zu denken. Der Krieg hatte die Welt in ein «Vorher» und «Nachher» gespalten, eine Spaltung, die von Wissenschaftlern

78 Freud, Sigmund: Zeitgemässes über Krieg und Tod. Gesammelte Werke. Hrsg. von Anna Freud. Frankfurt a. M., Fischer, 1969. Band 10, S. 324.

79 Bleuler 1919, S. 49. 
verschiedenster Disziplinen registriert und zum Teil schon damals bewusst wahrgenommen wurde. Die Spaltung wurde zur fachübergreifenden Metapher $^{80}$. Die Verlorenheit des Menschen, sein Kontaktverlust zum Früher und zu seinen Mitmenschen, sein «autistisches» Lebensgefühl zeigte sich auch in den Künsten ${ }^{81}$. Die Orientierungslosigkeit der Medizin in diesen Jahren wurde unterstrichen durch die grosse Grippeepidemie von 1918/19, der noch mehr Menschen zum Opfer fielen, als sie der Krieg gefordert hatte. Epidemie und Krieg werden bei Bleuler zu Metaphern, die in einem Atemzug genannt werden ${ }^{82}$. Dazwischen steht der Arzt mit seiner Verantwortung nicht nur für den einzelnen Patienten, sondern für die Gesellschaft. Bleulers Ruf nach einer «sozialen Medizin» ${ }^{83}$ resultiert aus der Erkenntnis, dass der Raum, in den die Medizin gestellt ist, grösser und unübersichtlicher geworden ist. Hier berührt sich seine Sicht des menschlichen Gehirns mit der persönlichen Erfahrung des Weltkriegs.

Es waren die vielen, in seinen Augen wirkungslosen prophylaktischen und therapeutischen Massnahmen gegen die Influenza, die Bleuler in diesen Jahren beobachten konnte, die ihn gegen seinen Stand aufbrachten und zu einem Kommentar herausforderten ${ }^{84}$. Psychiater und Neurologen waren für die durch Weltkrieg und Epidemie ausgelösten Vorgänge im Bewusstsein der Gesellschaft schon allein deshalb besonders empfänglich, weil die Arbeit im Grenzgebiet von Geist und Gehirn ihr Alltag war ${ }^{85}$. Aber auch, weil sich die Auseinandersetzung mit Spaltungssituationen in der Entwicklung ihrer

80 Monakow schrieb: «Die Diaschisis, welche der Krieg gesetzt hat, muss doch endlich auch überwunden werden.» Er setzt hier die Aufspaltung von Hirnfunktionen nach einem neurologischen Trauma («Diaschisis») zu dem Trauma des Ersten Weltkriegs in Beziehung. Vgl. dazu Jagella et al. (oben Anm. 48), S. 31ff.

81 Zum Beispiel bei Paul Klee (1879-1940), dessen Interesse an der organischen Entwicklung, zur Überwindung seiner Zerschneidungsphantasien, an Bleulers Suche nach «einer natürlichen Einheit» erinnert.

82 «But we still too often forget the metaphoric bridges between the body and the state, and remain oblivious to the physiologies of the imagination and their links to more tangible organizations» (Porter, Roy: Gout: Framing and Fantasizing Disease. In: Bulletin of the History of Medicine 68 (1994), S. 27).

83 Bleuler 1919, S. 193.

84 Op. cit. S. 56ff. In der Schweiz zählte man 24449 Grippetote zwischen Juli 1918 und Juli 1919. Vgl. dazu Mörgeli, Christoph: Die Grippeepidemie von 1918 in der Schweiz. In: Zeitschrift der Schweizerischen Diabetes-Gesellschaft 123 (1996/97), S. 13-19.

85 Man könnte sogar die These vertreten, dass gerade die sogenannten Neuropsychiater, die über Hirnanatomie und Hirnpathologie zur Psychiatrie gekommen waren, für diese Grenzfragen besonders sensibel waren. $\mathrm{Zu}$ den Neuropsychiatern muss auch Freud gerechnet werden; 1885 hielt er seine Probevorlesung an der Wiener Universität über «Die Muskelstrecken des Gehirns», um daraufhin zum Privatdozenten für Neuropathologie ernannt zu werden. Freud reagierte auf das Kriegsgeschehen, ähnlich wie Monakow und Bleuler, tief deprimiert. Im Jahr 1915 verfasste er eine Reihe kleinerer Abhandlungen, die unter dem Sammeltitel «Zeitgemässes über Krieg und Tod» erschienen. Vgl. dazu Gay (oben Anm. 35), S. 401. 
eigenen Spezialfächer wiederfand ${ }^{86}$. Auch für Eugen Bleuler bedeutete der Erste Weltkrieg den entscheidenden Einschnitt: sein wissenschaftliches Werk befasst sich nach 1918 nunmehr mit Fragen der Ethik, der Forensik und dem Versuch einer neuen Psychologie, die die biologischen Grundlagen des Denkens und Fühlens berücksichtigt. Es sind Werke, die sich so auffällig von den früheren Arbeiten unterscheiden, dass sie, nicht ohne Hintergedanken, als Bleulers «naturphilosophische Schriften» bezeichnet und zusammengefasst wurden ${ }^{87}$. In Bleulers Kritik am «autistisch-undisziplinierten Denken in der Medizin» sind wichtige Thesen dieses Spätwerks vorgezeichnet. Beim Lesen dieses in seinem Stil so ganz aus dem Rahmen seiner sonstigen Arbeiten fallenden Werks gewinnt man den Eindruck, als betreibe hier Bleuler auch eine Art Selbstanalyse. Es scheint, als ginge es ihm um die Befreiung auch von eigenen überholten Ansichten, Klischees, die seinen Ansprüchen einer neuen, umfassenden Vorstellung von Medizin und Gesellschaft nicht mehr genügen konnten. Bleulers Schrift vom «autistisch-undisziplinierten Denken in der Medizin» ist mehr als die Kritik an der Medizin seiner Zeit: sie ist Zivilisationskritik.

Bleuler stellt unter dem Begriff des autistischen Denkens subjektive Wechselbeziehungen zwischen Arzt und Patient in den Vordergrund. Er führt einen grossen Teil der medizinischen Praxis auf irrationale Vorgänge zurück, von der einzelne Wirtschaftszweige profitierten, ohne dass der allgemeinen Not abgeholfen werde. Damit wird er nicht nur zum Kritiker der Medizin, sondern auch zum Kritiker der Gesellschaft. Eine Verbindung, die zum Beispiel in seinem Angriff auf die «Medikamentengrossindustrie» evident wird. Es ist die Kritik an einem eindimensionalen Positivismusverständnis, das seit dem 19. Jahrhundert Naturwissenschaften und Gesellschaft geprägt hatte. Als Psychiater erhebt er hier zumindest indirekt den Anspruch der Heilung einer Krise der Medizin und einer gesamtgesellschaftlichen Krise. Das war etwas Neues. Besonders mit dem Aufkommen des positivistischen Denkens hatte sich die Medizin immer mehr auf die Wahl der richtigen Methode konzentriert und die Handelnden selbst, Arzt und Patient, dabei ausgeschlossen. Die Objektivität, die damit erreicht werden sollte, wird nach Bleulers Ausführungen in dem Moment eine Scheinobjektivität, wo sie die unbewussten, die «affektiven» Bedürfnisse von Arzt und Patient unberücksichtigt lässt. Die kritische Prüfung der eigenen Wahrnehmung,

86 Neurologie, Psychiatrie, Psychoanalyse, Psychologie, experimentelle Hirnforschung gerieten nach 1914 immer mehr zu Einzeldisziplinen.

87 Siehe Minkowski, Mieczysław: Zum Angedenken an Eugen Bleuler. In: Schweizer Archiv für Neurologie und Psychiatrie 46 (1941), S. 6. Minkowski spricht von «naturphilosophischen Schriften», weil er sowohl das revolutionäre als auch das abseitsstehende Element dieser späten Arbeiten Bleulers empfunden hat. 
die Bleuler anstrebt, erinnert nicht nur assoziativ an die Veränderung des Weltbilds, wie sie durch Albert Einsteins (1879-1955) Relativitätstheorie herbeigezwungen wurde ${ }^{88}$. Sie wurde bereits 1919 von den Natur- und Geisteswissenschaftlern gleichermassen in einer Weise registriert, wie sie Einstein selbst nicht für möglich gehalten hatte ${ }^{89}$. Für Karl Popper (1902-1994) wurde sie zum entscheidenden Anstoss, der seine Philosophie begründete. Das Jahr 1919 war sein Schlüsseljahr, in dem er sowohl die Freudsche Psychoanalyse als auch die Relativitätstheorie kennenlernte, wobei letztere für ihn als «Einsteinsche Revolution» im Rückblick die wichtigeren Anregungen hinterliess ${ }^{90}$ :

«Ob nun Einsteins Theorie wahr ist oder falsch - sie zeigt, dass Wissen im klassischen Sinne, gesichertes Wissen, Gewissheit, unmöglich ist.» ${ }^{91}$ (...) So kam ich, gegen Ende des Jahres 1919, zu dem Schluss, dass die wissenschaftliche Haltung die kritische war; eine Haltung, die nicht auf «Verifikationen» ausging, sondern kritische Überprüfungen suchte: Überprüfungen, die die Theorie widerlegen konnten; die sie falsifizieren konnten, aber nicht verifizieren. Denn sie konnten die Theorie nie als wahr erweisen.» ${ }^{92}$

Wenn Popper von einer Revolution schreibt, so meint er dieses in umfassender Hinsicht, er drückt damit das Interdisziplinäre, auf die Gesellschaft Zurückwirkende aus, das von der Relativitätstheorie ausging ${ }^{93}$. Egal, wie sehr

88 Besonders die Einstellung zur Aussagemöglichkeit von Begriffen wurde verändert; vgl. zum Beispiel Kar, Robert: Ein Konflikt zwischen Natur- und Denkgesetzen. Erkenntnistheoretische Folgerungen aus der speziellen Relativitätstheorie. In: Philosophia naturalis 4 (1957), S. 12-57.

89 Vgl. Elton, Lewis: Einstein, General Relativity and the German Press, 1919-1920. In: ISIS 77 (1986), S. 95-103. Anlässlich seiner Berufung an die Zürcher Universität 1909 wurde Einstein erstmals in der Presse erwähnt (NZZ vom 10.3.1909); vgl. Pais, Abraham: Ich vertraue auf Intuition. Der andere Albert Einstein. Heidelberg u. a., Spektrum, 1995, S. 185. Um 1914 bekam die allgemeine Relativitätstheorie ihre Form, aber erst 1919 wurde sie weltweit populär, weil die theoretisch vorhergesagte Lichtablenkung im Gravitationsfeld durch die Sonnenfinsternis auch «praktisch» bewiesen werden konnte. 1912 wurde Einstein erstmals für den Nobelpreis nominiert, den er erst 1922 erhielt.

90 Popper, Karl: Ausgangspunkte. Hamburg, Hoffmann \& Campe, 1995, S. 49.

91 Popper, Karl: Auf der Suche nach einer besseren Welt. München 1991,zit. nach: Karl Popper. Dargestellt von Manfred Geier. Hamburg, Rowohlt, 1994, S. 38.

92 Popper 1995, S. 48. Poppers Haltung, oft als krass positivistisch missverstandeń, ähnelt der Eugen Bleulers in der intensiven Beschäftigung mit dem Erkenntnisproblem in der Naturwissenschaft, mit der «Psychologie der Forschung». Wie Bleuler ging es ihm um das «Abgrenzungsproblem»; seine «3 Welten» sollten subjektive und objektive Tatsachen deutlicher voneinander trennen, wobei entscheidend war, dass sie dennoch aufeinander wirken konnten. Die kritische Einstellung gegenüber der Idee, mit naturwissenschaftlichen Methoden oder überhaupt mit irgendeiner geschlossenen Theorie zur Wahrheit vordringen zu können, liess Popper auch gegen Freuds Psychoanalyse reserviert bleiben. Sein spätes Buch «The self and its brain» (1977), zusammen mit John C. Eccles verfasst, thematisiert den Wandel des Erkenntnisproblems im 20. Jahrhundert. Popper war ein Bewunderer von Sokrates: dessen Ausspruch «Ich weiss, dass ich nichts weiss», hat Bleuler pragmatisch abgewandelt.

93 Am 7. November 1919 hiess es in der Times: «Wissenschaftliche Revolution. Neue Theorie des Universums. Newtons Vorstellungen umgestürzt» (zit. nach Pais, oben Anm. 89, S. 191). 
Einstein in mancher Hinsicht missverstanden wurde, er hatte ein Paradigma geschaffen, das zur kulturellen Umbruchszeit nach dem Ersten Weltkrieg passte $^{94}$. Entscheidend ist hier, wie dieses in der Gesellschaft und besonders im wissenschaftlichen Diskurs aufgenommen wurde. Die Rezeption des Begriffs ist hier wichtiger als die ursprüngliche szientifische Aussage. Eine der wichtigsten Botschaften der speziellen Relativitätstheorie war, dass der Zustand des Betrachters in die Erkenntnisfindung einbezogen werden muss und dass hierbei die Alltagserfahrung des Sehens und Beobachtens nicht unbedingt mit der wissenschaftlichen Wahrheit übereinstimmen muss ${ }^{95}$. Monakows Vorwurf an Bleuler in bezug auf die Unterscheidung von Vulgärpsychologie und biologischer Psychologie ist genau so zu verstehen. Die Unklarheit der Begriffe, das Oszillieren zwischen naturwissenschaftlichem und allgemein-kulturellem Anspruch machte diese Diskussion für die Medizin und besonders für Neurologie und Hirnforschung interessant $t^{96}$.

Einsteins vierdimensionale Raumzeit barg Momente der Befreiung in sich, die für eine um 1900 in die Sackgasse des Lokalisationsdenkens geratene Hirnforschung wie der lang ersehnte Ausweg erscheinen musste. So wie Einstein die Berücksichtigung neuer Faktoren für ein neues Weltbild forderte, so war auch das Bild vom menschlichen Gehirn, seiner Ausdehnung und Funktion, seiner Wechselbeziehung mit der Umwelt auf neue, dynamische Konzepte angewiesen. In der speziellen Relativitätstheorie wurde eine neue Definition der Zeit gesehen ${ }^{97}$, die zu den Zeitrelativierungen und einer Vorstellung von Gleichzeitigkeit passte, die Monakows Forschung implizierte. Einsteins «Skepsis gegen den realistischen Abbildcharakter der Begriffe», den «Begriffsdogmatismus einer aprioristischen Haltung» war Positivismuskritik immanent ${ }^{98}$. Einsteins Kritik galt nicht dem Positivismus als Methode, sondern der Vorstellung, er würde alle Phänomene erklären können. Begriffe hätten nur ihre Berechtigung im Kontext ihrer Erlebbarkeit, diese wiederum ist an das Subjekt gebunden. Wenn man Bleuler so versteht, so löst sich der scheinbare Widerspruch seiner Forderung, man solle systematisch und empirisch forschen und gleichzeitig die unbe-

94 Die Heroisierung Einsteins durch breite Massen wird im Zusammenhang mit dem Ersten Weltkrieg gesehen, «die Erschöpfung und das Chaos, die ein Jahr nach dem Ende des Ersten Weltkriegs herrschten, liessen Einsteins Auftritt noch dramatischer werden» (zit. nach Pais, oben Anm. 89, S. 194).

95 Vgl. Pais (oben Anm. 89), S. 194.

96 Vgl. dazu Janich, Peter: Über den Einfluss falscher Physikverständnisse. In: Das Gehirn Organ der Seele? Hrsg. von Ernst Florey und Olaf Breidbach. Berlin, Akademie, 1993, S. 312.

97 Vgl. Kar (oben Anm. 88), S. 19.

98 Zit. nach Müller-Markus, Siegfried: Erkenntnisprobleme der Relativitätstheorie. In: Philosophia naturalis 7 (1961), S. 184. 
wussten Anteile dieses Forschungsprozesses erfassen. Bleuler nennt 1919 den Zentrenbegriff aus der Hirnforschung als Beispiel für den «Autismus in der Begriffsbildung» und lässt dabei weitergehende Zusammenhänge aufleuchten:

«Noch früher hat man in der Physiologie des Zentralnervensystems den Begriff der Zentren geschaffen, der aus seiner alten Starre nun endlich zu beweglichem Leben erweckt wird.» ${ }^{99}$

Er verweist hier wiederum auf Monakow. Dieser hatte bereits 1905 und dann in ausgedehnter Form im Jahr 1914 «Die Lokalisation im Grosshirn» als ein solches mehrdimensionales Konzept dargestellt, in dem dem Faktor der Zeit im Sinne der «chronogenen Lokalisation» zentrale Bedeutung zukam ${ }^{100}$. Dass hier auch ein erkenntnistheoretisches Problem verborgen lag, auf das Neurologie und Hirnforschung zu reagieren hatten, zu dessen Lösung diese umgekehrt aber auch beitragen konnten, ist offensichtlich. Die Wissenschaftler begaben sich mit ihren dynamischen Konzepten der Hirnfunktionen - sei es nun für die Neurophysiologie oder für die Psychiatrie - auf ein Gebiet, das an philosophische Konsense der Gesellschaft des späten 19. Jahrhunderts angrenzte. Einer dieser Konsense war der des positivistischen Weltbilds, das der Realität des Alltags, gerade des Naturwissenschaftlers, eine gewisse Stabilität und Beschaulichkeit garantierte. Eben diese Scheinstabilität, die auf einer Scheinobjektivität ruhte, war es, die Bleuler 1919, in diesem Fall für die Medizin, erschüttern wollte. Bleulers neurologischer Gegenspieler in Zürich, Constantin von Monakow, nahm diese nicht auf den ersten Blick offenliegende Intention Bleulers in der oben besprochenen Rezension von 1920 auf und bezog sich dabei auf Einsteins Relativitätstheorie, wenn er fragte:

«Was ist nun Wahrheit?» ${ }^{101}$

99 Bleuler 1919, S. 71f.

100 «... um aber zu einer Lokalisation der Funktionen zu gelangen, dazu bedürfen wir - über die anatomischen Verbindungen hinaus - zunächst einer nach wissenschaftlichen Prinzipien (und nicht nur nach einer populären Betrachtungsweise) vorgenommenen Analyse der örtlichen Reiz- und Ausfallserscheinungen, wobei in weitestgehender Weise das zeitliche Moment (zeitlicher Aufbau der Funktion) zu berücksichtigen ist» (Monakow, Constantin von: Die Lokalisation im Grosshirn und der Abbau der Funktion durch kortikale Herde. Wiesbaden, Bergmann, 1914, S. 2).

101 «Gewöhnlich umschreibt man sie mit Übereinstimmung mit der Wirklichkeit. Was ist aber Wirklichkeit? Es gibt eine Wirklichkeit (Realität) rein subjektiver Natur (unsere Gefühle) und solche objektiver Natur, d.h. eine durch exterozeptive Beobachtung und durch Verifikation seitens Anderer erhärtete Wirklichkeit (Experiment, Rekonstruktion). Aber auch diese Wirklichkeit ist eine bedingte oder relative, d.h. durch etwaige persönliche Momente modifizierte (das bezieht sich sogar auf die physikalischen Experimente, Ausnahmen von der Regel: Relativitätslehre von Einstein)» (Monakow 1920, S. 173). 
Bleuler antwortete ihm 1921:

«Die Wahrheit ist etwas Relatives.» ${ }^{102}$

Das vormals wenigstens vordergründig einfache Schema der Arzt-PatientBeziehung ist für Bleuler ins «Schwanken» geraten wie der Zeitbegriff ${ }^{103}$. Er sieht seine Zeit als die «Zeit der schwindelnden Ausbildung der realistischen Kenntnisse und des realistischen Denkens», und als solche könne sie gar nicht «das affektiv dereierende Denken und das entsprechende Fühlen entbehren ${ }^{104}$. Hier wird deutlich, dass Bleulers Schrift über das «autistischundisziplinierte Denken in der Medizin» nicht als Streitschrift gegen irrationales Handeln zu verstehen ist, wie es in der Regel interpretiert wurde. Sie ist vielmehr ein Aufruf zur genauen und «bewussten» Trennung «irrationaler» und objektiver Elemente in wissenschaftlicher Forschung und Praxis und ebenso in der bewussten Wahrnehmung gesellschaftlicher Vereinnahmungen der Medizin. Patienten sind für Bleuler aktive Mitglieder in der diagnostischtherapeutischen Gemeinschaft. In ihrem irrationalen Bedürfnis nach Trost und Zuwendung verlören sie manchmal das Mass des Statthaften, und es sei die Aufgabe des Arztes, ihnen bei der Rückkehr in die Gesellschaft zu helfen. Davon wiederum könne diese nur profitieren.

Hinter Bleulers Kritik am gewissermassen regellosen und unkoordinierten Arbeiten der Ärzte steht ein starkes moralisches Moment. Die Berücksichtigung der eigenen psychischen Triebfedern im ärztlichen Alltag sollte nicht nur den Aspekten der Effizienz und des wissenschaftlichen Fortschritts dienen, sie sollte auch eine konkrete moralisch-sittliche Verbesserung der Bevölkerung bringen und damit der Gesellschaft Nutzen bringen ${ }^{105}$. In Bleulers Verständnis hat der Arzt eine sozial-sittliche Aufgabe, bei der das Wohl

102 Bleuler, Eugen: Naturgeschichte der Seele und ihres Bewusstwerdens. Berlin, Springer, 1921a, S. 323. Bleuler versucht im Kapitel «Lebens- und Weltanschauung» eine erkenntnistheoretische Beweisführung, in der die Erkenntnisse über «Raum und Zeit» zur «Realität» des Gehirns und seiner Funktionen in Beziehung gesetzt werden. Er folgert daraus, dass es unmöglich sei, «etwas Unendliches oder Absolutes mit unseren Vorstellungen und Erfahrungen in Verbindung zu bringen» (op. cit., S. 324). Vielleicht mit Blick auf die oben zitierte Äusserung Monakows fügt er hinzu: «Das Relativitätsprinzip Einsteins hat mit diesen Fragen nichts zu tun»; eine Erläuterung gibt er allerdings nicht. Das Werk endet mit der nochmaligen Feststellung: «Dabei weiss ich, dass die Wahrheit etwas Relatives ist. Für den jetzigen Stand unserer naturwissenschaftlichen Kenntnisse halte ich das Gesagte im grossen und ganzen für Wahrheit, nicht aber für die Wahrheit» (op. cit., S. 338).

103 Bleuler, Eugen: Mnemistische Biologie und Psychologie, 1936, zit. nach: Gedanken Eugen Bleulers. Hrsg. von Jakob Wyrsch. Zürich 1957.

104 Bleuler 1921a, S. 336. Er verwandte später den Ausdruck «dereierendes Denken» anstatt «autistisch» (Bleuler 1921, S. V).

105 Bleuler 1919, S. 173. Er macht diese Auffassung im Abschnitt «Vom medizinischen Autismus in der Alkoholfrage» exemplarisch deutlich (op. cit., S. 78-83). 
des einzelnen Patienten nur im Kontext der ganzen Gesellschaft betrachtet und abgewogen werden dürfe. Die in seinen Augen «autistische» Wechselbeziehung zwischen dem «brennende[n] Verlangen des Kranken, dass ihm Hilfe zuteil werde» und dem «Trieb des Arztes» ${ }^{106}$, diesem Wunsch so schnell als möglich nachzukommen, könne dieser übergeordneten Anforderung nicht in jedem Fall gerecht werden. Bleuler begibt sich auf einen problematischen und unübersichtlichen Weg, wenn er postuliert, der Arzt habe «die Tragweite seiner Anordnungen ebenso sehr vom allgemein-sozialen Standpunkt wie von dem individuellen eines bestimmten Kranken aus in Erwägung zu ziehen ${ }^{107}$.

\section{Schlussfolgerungen}

Die Vorstellung, dass «der Nachweis der örtlichen und zeitlichen und funktionellen Verbindung im nämlichen Hirn» ${ }^{108}$ vonnöten sei, um eine Wahrnehmung zu überprüfen, war das Ergebnis neuer Konzepte der Hirnforschung, wie sie um die Wende zum 20. Jahrhundert in Zürich aufkamen, um durch das Trauma des Ersten Weltkriegs bestärkt zu werden. Diese Vorstellung bewegte nicht nur die Hirnforschung selbst, indem sie vom Lokalisationsdenken des 19. Jahrhunderts wegführte, sie hatte weitertragende Folgen. Sie lief auf eine Änderung des Bewusstseins der Medizin hinaus. Medizinkritik entstand hier aus dem Zusammenspiel von wissenschaftlicher Beobachtung und vertiefter Wahrnehmung eines kulturellen Umbruchs.

Ernst Haeckels (1834-1919) «Welträthsel» von 1899 stehen für die Popularisierung einer neuartigen Diskussion um die Möglichkeiten und Grenzen der Erkenntnis, in der sich naturwissenschaftliche mit gesellschaftlichen Reflexionen verbanden ${ }^{109}$. Du Bois-Reymonds «ignorabimus» wurde in diesem Kontext von August Forel (1848-1931) in ein nur noch «vorläufiges <ignoramus» umgewandelt ${ }^{110}$. Mit Henri Bergsons (1859-1941) «l'évolution

106 Op. cit., S. 86.

107 Op. cit., S. 44. So finden sich mehrfach Verweise auf diese breitgefasste Verantwortung des Arztes als Arzt der Gesellschaft im Zusammenhang mit eugenischen Abwägungen. Vgl. Bleuler 1911.

108 Bleuler 1919, S. 154.

109 Haeckel, Ernst: Die Welträthsel. Gemeinverständliche Studien über Monistische Philosophie. Volksausgabe. Mit einem Nachworte: Das Glaubensbekenntnis der Reinen Vernunft. Stuttgart, Kröner, 1903 (1. Auflage: Bonn, E. Strauss, 1899).

110 Forel, August: Über unser menschliches Erkenntnisvermögen. Sonderdruck aus: Journal für Psychologie und Neurologie 21 (1915), S. 19. 
créatrice» wurde 1907 eine Positivismuskritik formuliert, die für Kultur- und Naturwissenschaftsgeschichte revolutionär wurde und die den Autor zum «intellektuelle[n] Star der Vorkriegsgeneration» ${ }^{11}$ machte. Weniger bekannt, aber gerade für die Neurowissenschaften ähnlich wichtig wie Bergson wurde der Arzt und Zoologe Richard Semon (1859-1918). Semons Konzept der «mneme» als «erhaltendes Prinzip im Wechsel des organischen Geschehens» von 1905 und Bergsons «élan vital» spiegeln das Motiv dynamischer Entwicklung bei gleichzeitiger Suche nach neuer Stabilität der Anschauungen, wie sie verstärkt nach 1919 Hirnforschung und Gesellschaft kennzeichneten. Monakows «hormé» und Bleulers «mneme» der Spätwerke beruhten auf der Auseinandersetzung mit Bergson und Semon, und sie sollten als neue Termini den Widerspruch zwischen Erkenntnis und Alltagserfahrung aufheben ${ }^{112}$.

Monakow beobachtete Regenerationsvorgänge im Gehirn, die nur in ihrer zeitlichen Abfolge verstehbar werden. Bei Bleuler war es die langjährige Begleitung schizophrener Patienten, vielleicht auch die Beobachtung veränderter Raum-Zeit-Wahrnehmung bei Schizophrenie, die ihn nach neuen Erklärungsmodellen suchen liess. Die Erweiterung des Zeitbegriffs und seine Verwendung bei der Beschreibung des Gehirns, also die Zusammenbringung räumlicher und zeitlicher Sphäre, wurde sowohl für Monakow als auch für Bleuler Anlass zur Entwicklung offener Krankheitsvorstellungen. Beide kamen zu dem Schluss, dass Gefühle oder Affekte in der naturwissenschaftlichen Forschung berücksichtigt werden müssten, obwohl sie zum Teil «über Raum und Zeit erhaben» wären ${ }^{113}$. Die scheinbar «unüberbrückbare Kluft» zwischen Moral und Gehirn wurde durch die Beachtung der gemeinsamen morphologisch-geschichtlichen Entwicklung verstehbar ${ }^{114}$.

Bleulers Behauptung, dass die Objektivität selbst des Experiments oder der Statistik immer an das Subjekt des Forschers gebunden ist und damit emotionale, unbewusste Anteile sowohl in Aufbau als auch Durchführung und Ergebnisinterpretation des Forschers einfliessen, sprengte den auf der

111 Weinmann, Martin, in: Metzler Philosophen Lexikon. Hrsg. von Bernd Lutz. Stuttgart 1989, S. 99. Bergsons «Durée et simultanéité» von 1922 war eine Auseinandersetzung mit Einsteins Relativitätstheorie, in der er «ganz unschulmässig ... die Chance zur Selbstverständigung und Selbstkorrektur ergriffen hat» (op. cit., S. 100).

112 Hormé [gr.]: Antrieb, von: in Bewegung setzen, gr. «Hormé» und «mneme» sollten den herkömmlichen Seelenbegriff ersetzen, auf biologisch-physiologischer Basis. Beide «schliessen die Vorstellung von Engrammen als biologische Gedächtnisäquivalente in sich ein» (Jagella et al., oben Anm. 48, S. 46).

113 Monakow, Constantin von: Gefühl, Gesittung und Gehirn. Wiesbaden, Bergmann, 1916, S. 120.

114 Op. cit., S. 116. 
Anerkennung des Positivismus beruhenden Konsens der Medizin seiner Zeit. Seine Auseinandersetzung mit der Psychoanalyse war hierfür sicherlich entscheidend. Andererseits wollte er keine Rückwendung zur Mythologisierung der Psyche. Die empirische Erfahrung sollte erweitert werden durch die aus der Psychoanalyse gewonnene Erkenntnis, dass jede Handlung, auch wenn sie von experimentellen Umständen abgesichert ist, nicht als nur rational verstanden werden kann, da für ihn das Gehirn als eine Einheit funktionierte.

Die Nichtanerkennung der Psychologie als Wissenschaft führt Bleuler direkt zum Kernproblem medizinischer Forschung überhaupt: es seien die gleichen Gründe, die zur Ablehnung der Psychologie führen, die auch den Aufbau einer blossen Scheinobjektivität unterhalten. Das Kriterium der «Greifbarkeit» von Fakten, das der modernen Naturwissenschaft oberstes Gebot wurde, habe die Psychologie aus dem Kontext der verschiedenen empirischen und experimentellen Versuchsansätze ausgeschlossen, und zwar aufgrund eines Unterschiedes, der als prinzipiell angenommen wurde, dem Unterschied zwischen Geist und Körper ${ }^{115}$. «Wissenschaftliches Denken» im Sinne Bleulers ist «kausalverstehendes Denken» ${ }^{116}$. Es handelt sich für ihn hier aber um keine Synthese beider Anteile, sondern Kausalität und Verstehen sind eine Einheit, wie das Gehirn und die aus ihm entstehenden Funktionen des individuellen und sozialen Lebens eine Einheit sind. Diese Differenzierung ist sehr wichtig, da hierdurch deutlich wird, dass Bleulers Positivismuskritik nicht gegen positivistisches Schauen an sich gerichtet ist: wenn Geist und Materie im Gehirn eine Einheit bilden, so ist die Beschäftigung mit der Psyche eine Angelegenheit der Naturwissenschaft. Hier offenbart sich Bleulers fundamentale Differenz zu Freud: eine exklusive Sicht der Psychoanalyse gibt es für Bleuler nicht, es gibt keinen eigenen Weg der Psychologie. Wohl aber muss die Sicht der Naturwissenschaft erweitert werden. Das «autistisch-undisziplinierte Denken in der Medizin» wurde auch im Blick auf diese Kontroverse mit Freud geschrieben, zu einem Zeitpunkt, als das Zerwürfnis beider Wissenschaftler offensichtlich geworden war ${ }^{117}$.

Ein Zugang zu den unbewussten Anteilen positivistischer Wissenschaft führt für Bleuler über die Hypnose, mit ihr könne man den Gefahren

115 Bleuler zitiert in diesem Zusammenhang Karl Jaspers Differenzierung von der Psychologie als verstehender Wissenschaft im Gegensatz zu den anderen medizinischen Disziplinen, die kausal seien. Gerade diese Scheindifferenzierung führe aber auch zur ungenügenden Effizienz und Erkenntnistauglichkeit der sogenannten exakten Wissenschaften. Vgl. Bleuler 1921, S. $132 \mathrm{ff}$.

116 Op. cit., S. 187.

117 Vgl. Harms (oben Anm. 42), S. VIII. 
autistischen Denkens in der Medizin besser begegnen. In der besonderen Bedeutung, die er der Hypnose für die Erkenntnistheorie der Medizin beimass, fand Bleuler Unterstützung bei August Forel, der um 1900 intensiv mit Suggestion und Hypnose als Therapie für «Psyche» und «Körper» experimentierte. Ähnlich wie Bleuler nimmt Forel die Angriffe aus der Ärzteschaft, die sich gegen die Aufnahme der Hypnose in die Allgemeintherapie verwahrte, zum Anlass, das Verhältnis der Medizin zu ihrer eigenen Methodik zu beleuchten ${ }^{118}$. Auch für ihn sind Materie und Seele eine Einheit, die durch das lebende Gehirn definiert ist, und jegliche Trennungsversuche von seiten der «Materialisten» oder «Spiritualisten» - die wiederum oft «unbewusst» vonstatten gingen - würden durch die Tatsache ad absurdum geführt, dass «wir nichts als Verhältnisse mit unserem Gehirn durch das Symbol unserer Sinne ergründen». Die Erfahrung der Hypnose zeige, dass Objektivität und Subjektivität «überhaupt blos in einem relativen Gegensatze» begriffen seien ${ }^{119}$. Die ganze Wissenschaft beruhe eigentlich nur auf «verglichenen Introspektionen» ${ }^{120}$. Die «reine Objektivität» sei eine pure Illusion, weil der Arzt «in seinen Ansichten stets von den Empfindungen, d. h. von der Gehirn-Reaction des Kranken» beeinflusst werde ${ }^{121}$. Wie Bleuler verbindet Forel seine Kritik an der Missachtung der Psychologie mit einer deutlichen Gesellschaftskritik: eine vernünftige «Socialpolitik» sollte die Ärzte aus der Zwickmühle ihrer «Gewinnsucht» befreien; ebenso prangert er die «kritiklose Anpreisung von chemischen Heilmitteln» an ${ }^{122}$. Der absolute Wahrheitsanspruch der Medizin der 2. Hälfte des 19. Jahrhunderts wird bei Bleuler wie bei Forel in Frage gestellt. Anders als Forel geht Bleuler aber vom praktischen Alltag des Mediziners aus und zeigt so die direkte Verbindung von der Theorie in die Praxis ärztlichen Handelns. Hierin ist der Grund für die Popularität seiner Medizinkritik zu sehen.

So wie der Autismus ein Zeichen des Ich-Zerfalls, der Ich-Spaltung ist, so wäre mit Bleuler eine Spaltung der Einheit von Gefühl und Denken absurd und irreführend. Eine Wissenschaft, die diese «natürliche Einheit» leugne, wie sie auch die Einheit von Biologie und Psyche leugne, könne nicht wirklich ernstgenommen werden. In ihrer Ausblendung affektiver Elemente beim Naturwissenschaftler selbst werde sie nicht objektiv und seriös, wie sie

118 Forel, August: Suggestionslehre und Wissenschaft. In: Zeitschrift für Hypnotismus 1 (1892), S. $1-10$

119 Op. cit., S. 7.

120 Forel, August: Der Hypnotismus oder die Suggestion und die Psychotherapie. 8. und 9. Auflage. Stuttgart, Enke, 1919, S. 11.

121 Forel, August: Der Hypnotismus in der Hochschule. Sonderdruck aus der Zeitschrift für Hypnotismus 4 (o. J.), S. 6.

122 Op. cit., S. 8. 
das vorgibt, sondern das Gegenteil, nämlich unglaubwürdig. Autistisches Denken zu überwinden, das wird für Bleuler zur Aufgabe des 20. Jahrhunderts und bedeutet zugleich den Abschied vom cartesianischen Dualismus. Das Erlebnis des Weltkriegs mit Verlust und Zerfall des Früher, früherer Konzepte der Medizin und menschlichen Zusammenlebens, sollte durch Einheitsbestrebungen im umfassendsten Verständnis aufgelöst und überwunden werden ${ }^{123}$.

Es scheint berechtigt, von einem Zürcher Konzept über «ratio et experientia» zu sprechen. Ein besonderes Klima der interdisziplinären Forschung von Neurologie und Psychiatrie beförderte hier die alte Auseinandersetzung um die Grenze zwischen Geist und Materie neu und dehnte sie auf die Frage nach den Grenzen der Medizin aus. Unter dem Einfluss der Freudschen Psychoanalyse und in der langjährigen Tradition eines neurologischpsychiatrischen Kolloquiums wurden in Zürich Fragen erörtert, die nicht nur die Definition der eigenen Fachdisziplin betrafen, sondern über diese hinausgehend auch Fragen der Erkenntnistheorie, der Ethik und Moral behandelten.

Die Frage nach dem richtigen Verständnis von Neurologie und Psychiatrie wurde hier mit der generellen Frage nach der Haltung zur Naturwissenschaft des 20. Jahrhunderts verbunden. Bleuler und Monakow versuchten einen neuen Weg zu gehen, indem sie trotz tiefgreifender Verschiedenheiten in der Bewertung biologischer Prozesse die Frage nach den Grenzen zwischen Psyche und Soma mit der Frage nach den Grenzen des Erkennens verknüpften. Bleulers kaum beachtete Relativierung des Krankheitsbegriffs, wie er sie zunächst am Beispiel der Schizophrenie vornahm und dann 1919 auf den Zustand der Medizin extrapolierte, beruhte auf der Berücksichtigung des zeitlichen Ablaufs der Funktionen:

«Ich halte es aber für noch wichtiger als die Abgrenzung der Krankheit nach aussen, dass einmal klar ausgesprochen worden ist: innerhalb dieser Gruppe kennen wir noch keine natürlichen Grenzen; was man bis jetzt für Grenzen ausgab, sind Grenzen von Zustandsbildern, nicht von Krankheiten.»124

Der Ideenaustausch über die «Lokalisation im Grosshirn» wurde zur Standortbestimmung des eigenen Fachs und gleichzeitig zur Standortbestimmung der Medizin zu Beginn des 20. Jahrhunderts.

123 Vgl. Harrington, Anne: A Feeling for the "Whole". The holistic reaction in neurology from the fin de siècle to the interwar years. In: Fin de siècle and its legacy. Hrsg. von Micul Teich. Cambridge, University Press, 1990.

124 Bleuler 1911, S. 229. 